Application Note

\title{
The network makeup artist (NORMA-2.0): Distinguishing annotated groups in a network using innovative layout strategies
}

\author{
Evangelos Karatzas ${ }^{1, *}$, Mikaela Koutrouli ${ }^{2, *}$, Fotis Baltoumas ${ }^{1}$, Georgios A. Pavlopoulos ${ }^{1,3, *}$ \\ ${ }^{1}$ Institute for Fundamental Biomedical Research, Biomedical Sciences Research Center "Alexander Fleming", Vari, Greece \\ ${ }^{2}$ Novo Nordisk Foundation Center for Protein Research, Faculty of Health and Medical Sciences, University of Copenhagen \\ ${ }^{3}$ Center for New Biotechnologies and Precision Medicine, School of Medicine, National and Kapodistrian University of Athens, \\ 11527, Athens, Greece
}

*To whom correspondence should be addressed

\begin{abstract}
Motivation: Network biology is a dominant player in today's multi-omics era. Therefore, the need for visualization tools which can efficiently cope with intra-network heterogeneity emerges.
\end{abstract}

Results: NORMA-2.0 is a web application which uses efficient layouts to group together areas of interest in a network. In this version, NORMA-2.0 utilizes three different strategies to make such groupings as distinct as possible while it preserves all of the properties from its first version where one can handle multiple networks and annotation files simultaneously.

Availability: The web resource is available at http://norma.pavlopouloslab.info/. The source code is freely available at https://github.com/PavlopoulosLab/NORMA.

Contact: pavlopoulos@fleming.gr

\section{Introduction}

Network visualization is important to capture patterns and understand the associations among various biomedical entities coming from different repositories $(1,2)$. To this end, several visualization tools have been proposed (3). However, despite their richness in the interactivity and variety of analysis options they offer (e.g., Cytoscape's apps (4)), only few of them such as Cytoscape (5) or Arena3D $^{\text {web }}$ (6) can cope with node heterogeneity.

In this article, we present a major update of the NORMA web application (7) which now incorporates three different layout strategies to make annotated groups or areas of interest in a network more visually distinct. This way, NORMA-2.0 offers options for more appealing representations and enables a more targeted knowledge extraction and storytelling.

\section{Methods}

\subsection{General functionality}

NORMA is a handy web tool for interactive network annotation, visualization, and topological analysis through which users are able to handle multiple networks and annotations simultaneously. It offers group highlighting with the use of shaded areas (convex hulls) or pie-chart-like nodes while it comes with a functionality for directly comparing several networks topologically as well as different group annotations for the same network. In addition, users can perform clustering analysis on-the-fly and highlight nodes using any custom color scheme (e.g., expression values).

\subsection{Custom Layouts}

In this version, NORMA-2.0 utilizes three different strategies to visually separate annotated areas of interest in a network in combination with established layout algorithms, offered by igraph (8).

\subsection{Implementation}

NORMA-2.0 is written in R/Shiny. Network visualization is offered by d3.js. The 3D network and convex hull visualizations are offered by plotly. 
bioRxiv preprint doi: https://doi.org/10.1101/2022.03.02.482621; this version posted March 4, 2022. The copyright holder for this preprint (which was not certified by peer review) is the author/funder, who has granted bioRxiv a license to display the preprint in perpetuity. It is made

\section{Karatzas et al.} available under aCC-BY-NC-ND 4.0 International license.

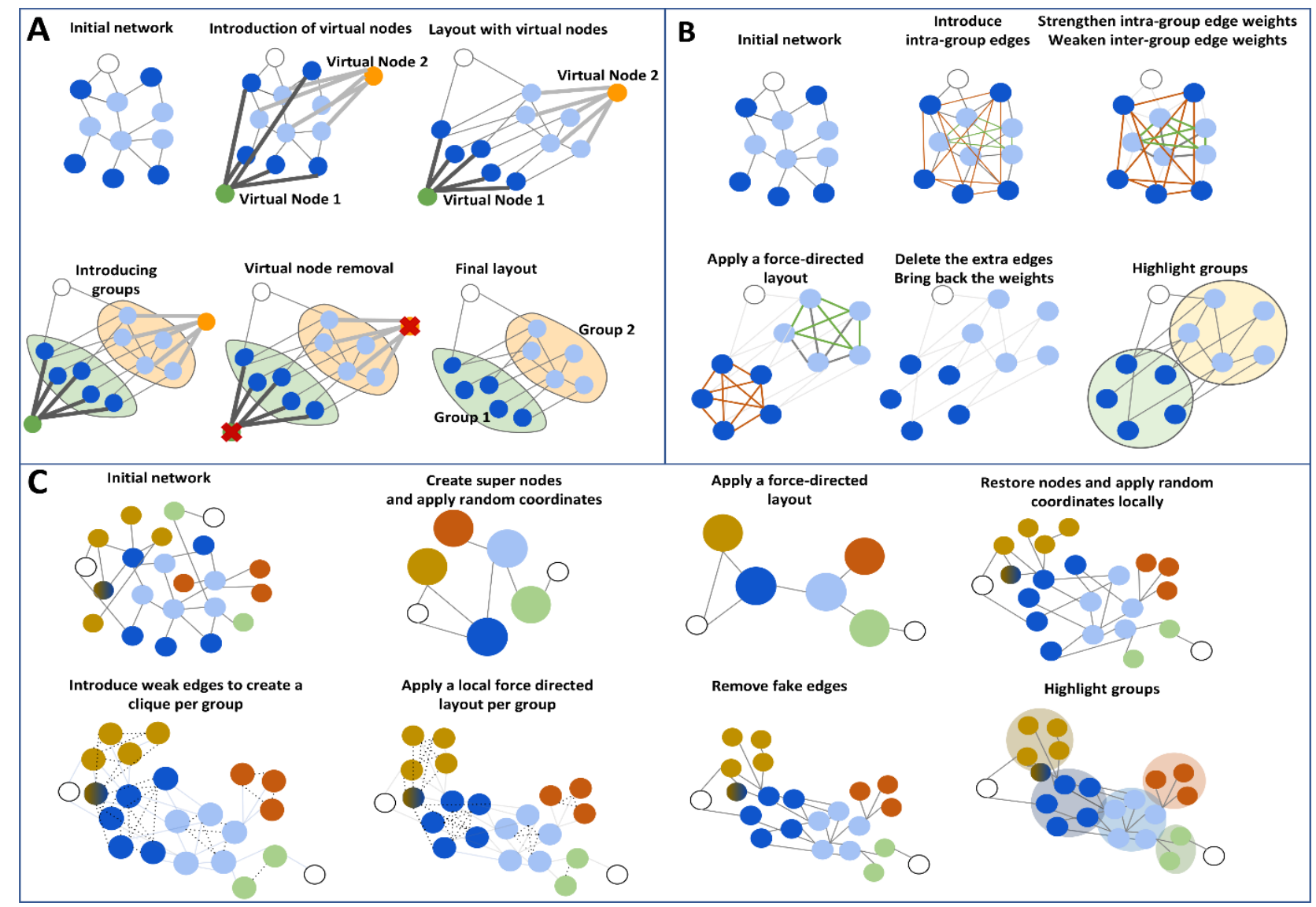

Figure 1. Three different layout strategies to highlight areas of interest within a network and make them as distinct as possible.

Strategy 1 - Virtual nodes: As in the original version, NORMA-2.0 introduces one virtual node per group which behaves as a hub. Upon creation, edges with heavy weights are assigned to this node, linking it with all nodes from the same group. Then, any traditional layout algorithm can be utilized until it converges. The difference compared to directly applying a layout algorithm on the network is that the virtual nodes will attract the group-specific nodes as if they were parts of the network. After completing the layout execution, all virtual nodes are removed (Figure 1A).

Strategy 2 - Gravity: Here, NORMA-2.0 introduces intra-group edges where necessary to generate clique-like subnetworks (all-vs-all connections). As a second step, the intra-group edge weights are significantly increased whereas the inter-group edge weights are simultaneously decreased. Then, any of the offered layouts can be applied to adjust node coordinates. The introduced edges and weights only exist for the calculation of the layout coordinates, and do not carry over to the final visualized network (Figure 1B).

Strategy 3 - Super nodes: In this scenario, NORMA-2.0 introduces 'super-nodes' to represent each of the uploaded annotation groups. Then, it connects these groups with edges which correspond to the connections from the initial network. For example, if node A belonging to the annotation group 1 is connected to node B from group 2, then the group 1 super-node will also be connected to the group 2 super-node. Notably, the network becomes significantly smaller both in terms of node and connection numbers. In a second step, any of the available layouts can be applied on the 'super-network'. Upon layout convergence, all centroid coordinates of these super-nodes as well as the coordinates of no-group nodes can be further repelled according to a user-defined input. Then, all initial nodes will be placed around their respective super-nodes according to a second user-selected local layout choice. If a node belongs to more than one group, the average values for their $(x, y)$ coordinates are used for the final visualization (Figure 1C).

\section{ACKNOWLEDGEMENTS}

We would like to acknowledge support from the Hellenic Foundation for Research and Innovation (H.F.R.I) under the "First Call for H.F.R.I Research Projects to support faculty members and researchers and the procurement of high-cost research equipment grant", Grant ID: 1855-BOLOGNA. GAP was also supported by the project 'The Greek Research Infrastructure for Personalised Medicine (pMedGR)' (MIS 5002802), which is implemented under the Action 'Reinforcement of the Research and Innovation Infrastructure', funded by the Operational Program 'Competitiveness, Entrepreneurship and Innovation' (NSRF 2014-2020) and co-financed by Greece and the European Union (European Regional Development Fund). 
bioRxiv preprint doi: https://doi.org/10.1101/2022.03.02.482621; this version posted March 4, 2022. The copyright holder for this preprint

(which was not certified by peer review) is the author/funder, who has granted bioRxiv a license to display the preprint in perpetuity. It is made available under aCC-BY-NC-ND 4.0 International license.

\section{References}

1. Koutrouli,M., Karatzas,E., Paez-Espino,D. and Pavlopoulos,G.A. (2020) A Guide to Conquer the Biological Network Era Using Graph Theory. Front Bioeng Biotechnol, 8, 34.

2. Baltoumas,F.A., Zafeiropoulou,S., Karatzas,E., Koutrouli,M., Thanati,F., Voutsadaki,K., Gkonta,M., Hotova,J., Kasionis, I., Hatzis,P., et al. (2021) Biomolecule and Bioentity Interaction Databases in Systems Biology: A Comprehensive Review. Biomolecules, 11, 1245.

3. Gehlenborg,N., O’Donoghue,S.I., Baliga,N.S., Goesmann,A., Hibbs,M.A., Kitano,H., Kohlbacher,O., Neuweger,H., Schneider,R., Tenenbaum,D., et al. (2010) Visualization of omics data for systems biology. Nat. Methods, 7, S5668.

4. Saito,R., Smoot,M.E., Ono,K., Ruscheinski,J., Wang,P.-L., Lotia,S., Pico,A.R., Bader,G.D. and Ideker,T. (2012) A travel guide to Cytoscape plugins. Nat. Methods, 9, 1069-1076.

5. Shannon,P., Markiel,A., Ozier,O., Baliga,N.S., Wang,J.T., Ramage,D., Amin,N., Schwikowski,B. and Ideker,T. (2003) Cytoscape: a software environment for integrated models of biomolecular interaction networks. Genome Res., 13, 2498-2504.

6. Karatzas,E., Baltoumas,F.A., Panayiotou,N.A., Schneider,R. and Pavlopoulos,G.A. (2021) Arena3Dweb: interactive 3D visualization of multilayered networks. Nucleic Acids Res, 49, W36-W45.

7. Koutrouli,M., Karatzas,E., Papanikolopoulou,K. and Pavlopoulos,G.A. (2021) NORMA: The Network Makeup Artist - A Web Tool for Network Annotation Visualization. Genomics Proteomics Bioinformatics, 10.1016/j.gpb.2021.02.005.

8. Gabor Csardi and Tamas Nepusz (2006) The igraph software package for complex network research. InterJournal, Complex Systems, 1695. 
in
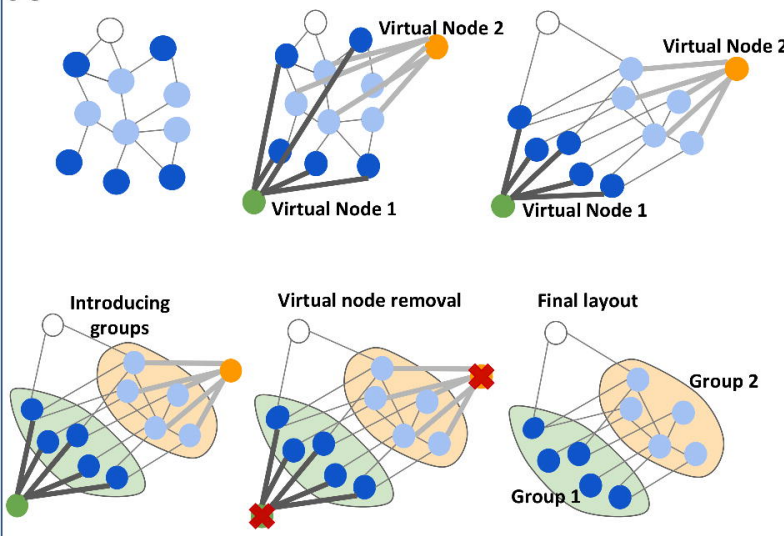

C

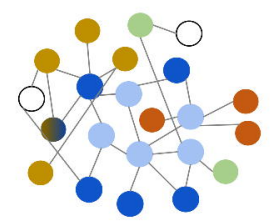

Introduce weak edges to create a clique per group
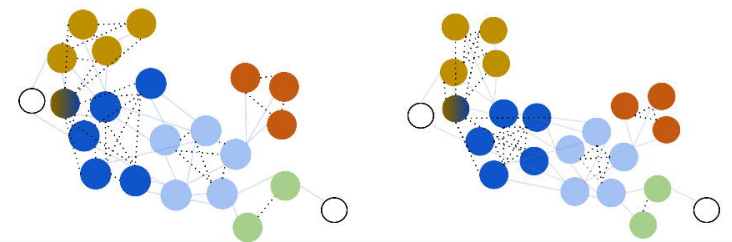

B Initial network

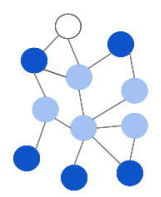

Apply a force-directed

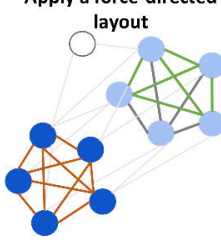
intra-group edges

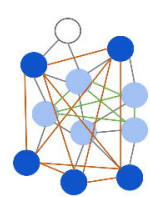

weights

Weaken inter-group edge weights
Delete the extra edges Bring back the weights

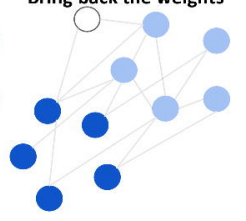

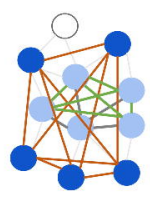

Highlight groups

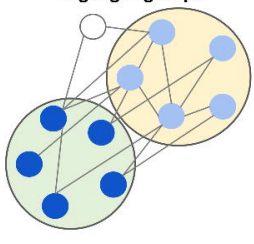

Apply a force-directed layout and apply random coordinates

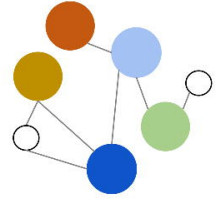

Apply a local force directed layout per group
Restore nodes and apply random coordinates locally
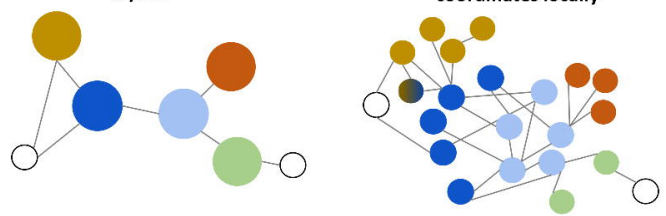

Highlight groups

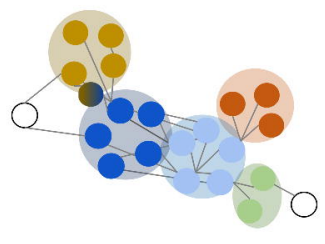

